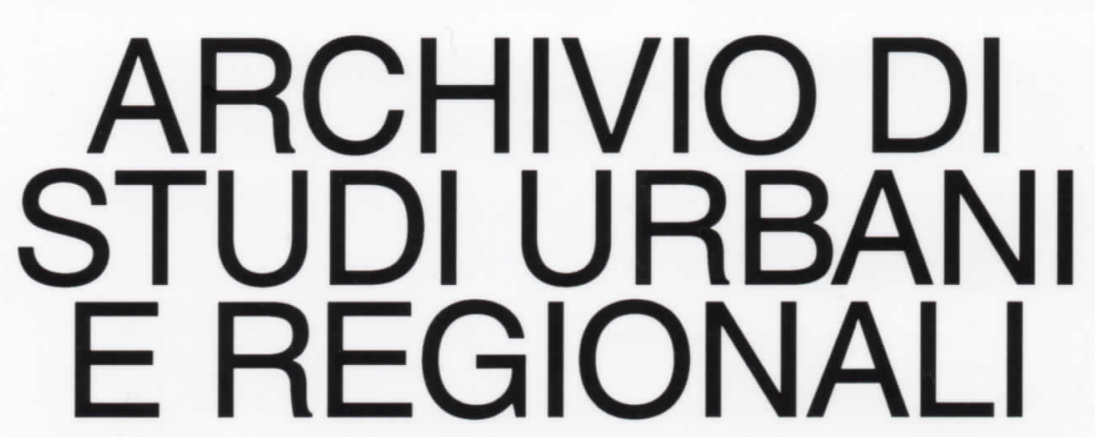

Franco Angeli - anno. XLVIII, n.123, 2018 


\section{SOMMARIO, A. XLVIII, N.123, 2018}

Fabio Andreassi, Il ruolo dei disastri naturali e dell'azione pubblica nella destrutturazione dell 'immaginario collettivo della città

Rosaria Battarra, Carmela Gargiulo, Rosa Anna la Rocca, Laura Russo, L'applicazione del paradigma smart city in Italia. Luci ed ombre delle sperimentazioni nelle città metropolitane

Francesco Gastaldi, Federico Camerin, Verso un censimento delle aree militari in Veneto

Mario Paris, Antonio Casella, Quale cooperazione intercomunale fuori dalle città metropolitane? Il percorso di riconoscimento dell'IPA veronese come laboratorio per il governo dell'area vasta

Anna Richiedei, Anna Frascarolo, Evoluzioni di un piano di recupero: quartiere gentrificato o multiculturale? Il caso del Carmine a Brescia

Dino Borri, Domenico Camarda, Nicola Schingaro, Maria Rosaria Stufano Melone, Percorsi cognitivi dello spazio per il supporto decisionale: alcune note a partire da casi di studio Maurizio Carta, Barbara Lino, Marilena Orlando, Innovazione sociale e creatività. Nuovi scenari di sviluppo per il territorio sicano

\section{Recensioni}




\title{
Quale cooperazione intercomunale fuori dalle città metropolitane? Il percorso di riconoscimento dell'IPA Veronese come laboratorio per il governo dell'area vasta ${ }^{1}$
}

\begin{abstract}
Mario Paris* e Antonio Casella**
Il paper affronta il tema della governance di scala vasta e delle forme di cooperazione fra Comuni negli ambiti al di fuori delle città metropolitane. Le riflessioni si misurano sulle questioni del cosiddetto "rescaling of formal planning activities" presenti in diverse normative nazionali e sono sviluppate a partire dai temi emersi nel percorso di definizione di un partenariato tra soggetti pubblici e privati nel Veronese che opera ad una scala intermedia fra quella municipale e regionale.

Parole chiave: Metropolizzazione; Governance metropolitana; Cooperazione intercomunale; Regione Veneto; Intesa Programmatica d'Area (IPA)

Which intermunicipal cooperation without città metropolitane? The process of recognition of IPA Veronese as testing ground for the governance of the intermediate scale.

The aim of this paper is exploring the intermunicipal governance through volunteer cooperation for those areas which are marked by mature metropolisation processes without a recognition by specific bodies or institutions (as città metropolitane in Italy). Focusing on the case of the IPA Veronese - a peculiar figure which involved public bodies and private companies in local government - we reflect about the intermediate scale and its role as role of testing ground for innovations in governance.
\end{abstract}

Key-words: Metropolisation processes; Metropolitan governance; Intermunicipal cooperation; Veneto Region; Intesa Programmatica d'Area (IPA)

\section{Introduzione}

Questo contributo si inserisce tra le riflessioni svolte all'interno della disciplina della pianificazione territoriale negli ultimi quindici anni in merito alle riforme istituzionali necessarie per poter comprendere e incidere sulle dinamiche spaziali contemporanee (Pugalis e Townsend, 2013; Healey, 2010; 2007; Bobbio, 2002). Si tratta di un campo di studi in cui è diventato necessario ripensare gli spazi d'azione per planners, policy-maker e tecnici,

\footnotetext{
${ }^{1}$ Inviato il 11 lug. 2016; nella forma rivista il 1 lug. 2017; accettato il 20 nov. 2017.

*Urb\&Com Lab., DAStU - Politecnico di Milano. mario.paris@ @olimi.it

** Istituto Commercio Servizi - ICS. info@ istitutocommercioservizi.org

Il presente saggio è frutto del lavoro congiunto dei due autori, che insieme hanno redatto

l'introduzione e le conclusioni. A. Casella ha curato il § 1, mentre M. Paris i § 2 e 3.
} 
anche attraverso la definizione di ambiti sub-regionali discreti (Fernàndez e Rubiera, 2012; Gualini, 2001; Bielli e La Bella, 1982) utili a governare le scale intermedie e non istituzionali delle dinamiche territoriali correnti (Brenner, 2003). L'obiettivo di queste operazioni, dovrebbe essere quello di raccordare processi in corso e forme di governance, recuperando la relazione fra istituzione e territorio (Balducci e al., 2017a). La nostra ricerca, ancorata al contesto italiano, riconosce la presenza di un buon numero di studi sui temi della gestione dei servizi, della razionalizzazione delle risorse e dell'amministrazione delle funzioni fondamentali a scala metropolitana (fra gli altri: Balducci e al., 2017b; Perulli, 2014; Vandelli, 2014 e 2012; Marotta e Pastena, 2013; Balducci e al. 2011; Ferrari e Galeone, 2010; Hulst e Van Montfort, 2007). Al contempo, evidenzia la relativa scarsità di riflessioni rispetto alle domande manifestate da Comuni, Enti Locali e forze sociali sulla necessità di orientamento e governance alla scala inter-municipale fuori dal contesto delle città metropolitane.

Sulla scorta di questo quadro teorico, in questo contributo siamo partiti da alcune pratiche recenti legate al tema della gestione dell'intercomunalità (Messina, 2011) e alla necessità di ripensare forme di governo dell'area vasta che supportino la costruzione di scenari strategici e visioni condivise oltre alla gestione amministrativa delle competenze, soprattutto negli ambiti delle città medie. In molti casi la richiesta da parte degli enti e degli attori economici è quella di far assumere all'area vasta il ruolo di laboratorio di innovazione nel governo del territorio, orientato alla creazione di sviluppo territoriale sostenibile e promozione di complementarietà fra asset socioeconomici locali, così come tracciato nelle recenti politiche di coesione territoriale dell'Unione Europea, introdotte dalla European Spatial Development Perspective del 1999 e riconfermate con il Trattato di Libsona (Meijers, 2012). Il campo d'analisi di questo contributo è il contesto italiano dove questa esigenza è ancor più marcata poiché l'attuale assetto istituzionale, non prevede figure specifiche su questi temi, salvo per quella delle città metropolitane previste dalla legge n. 56/2014 (Legge Delrio). Per questa ragione, come segnalato da Corò e Dalla Torre (2016), in ambito nazionale la sperimentazione di forme di innovazione fuori dagli ambiti riconosciuti come città metropolitane deve ricorrere a processi pragmatici, che possano includere anche il contributo delle istituzioni economiche, per capire il potenziale e i rischi connessi alle pratiche cooperative (Servillo e Lingua, 2014) e/o interattive (Torfing e al., 2012). L'utilizzo di queste pratiche come prove di innovazione potrebbe portare, a nostro giudizio, a sperimentare forme diverse di governance territoriale, più vicine alla scala e alla condizione contemporanea dei fenomeni territoriali, e più efficaci nell'intervenire su di essi.

Obiettivi. L'obiettivo di questo studio non è, quindi, quello di proporre una riflessione esaustiva sul tema della riforma della governance territoriale 
in Europa, né tantomeno di semplificare il ricco dibattito che negli ultimi anni si è sviluppato su questo argomento (fra gli altri: Knieling e Othengrafen, 2015; Reimer e al., 2014; Glassom e Marshall, 2010; Healey, 2010). Più semplicemente, ci proponiamo di verificare quale sia il potenziale di un approccio alternativo alle forme istituzionali di governo dell'area vasta per quei territori non riconosciuti come parti di città metropolitane ma che necessitano di strumenti di coordinamento su temi e problemi condivisi a livello locale. Per farlo, proponiamo la lettura critica di un'esperienza recente, quella della costituzione dell'IPA Veronese ${ }^{2}$ e l'identificazione di temi di riflessione utili alla definizione di nuove forme di governance territoriale. Si tratta di un percorso sviluppato con l'individuazione di alcune tematiche centrali nelle pratiche legate alla pianificazione strategica (fra gli altri: Balducci e al., 2011; Hartman e al., 2011; Albrechts, 2004) e al regional design (Balz e Zonneveld, 2015). In questo approccio le capacità di identificare attori, esplorare spazi di dialogo e definire le condizioni di interazione sono attività che hanno lo stesso peso delle azioni e i risultati che derivano dall'incontro degli agenti attivi sui territori. Al tempo stesso, questi sono stati adattati alla scala inter-comunale in un contesto di metropolizzazione matura. Nell'ambito del lavoro, la volontà di cooperare manifestata "dal basso" da parte di alcuni attori pubblici locali (sia politici, sia tecnici) si è concretizzata in una proposta orientata all'innovazione delle forme di governance sovracomunale e alle modalità di costruzione e implementazione dei progetti a questa scala.

Metodologia. La riflessione proposta muove da un'esperienza di ricerca applicata, realizzata attraverso il ricorso a due distinte tipologie di analisi. La prima, comparativo-descrittiva, utilizzata per analizzare le più diffuse forme istituzionali di governo dell'area vasta in ambito italiano e per approfondire gli aspetti strategici delle diverse opzioni valutandone opportunità e limiti. Una volta individuata la forma dei patti territoriali come uno degli assetti in grado di favorire la costruzione di governance territoriali flessibili e operative, ci siamo concentrati sullo studio di un caso specifico attraverso una seconda tipologia di analisi-osservazione empirica dei processi di costruzione e sviluppo dell'esperienza dell'Intesa Programmatica d'Area Veronese, della quale sono stati messi in evidenza opportunità e rischi, così da arricchire e supportare la riflessione sulle forme di governance attraverso la definizione di alcuni temi emergenti.

2 Gli autori di questo articolo, membri dell'Istituto Commercio e Servizi, hanno partecipato al percorso di costituzione dell'IPA Veronese quali consulenti dei Comuni di Verona e Valeggio sul Mincio (VR). Attraverso la loro ricerca, hanno supportato la segreteria tecnica dell'IPA Veronese e fornito materiali utili alla redazione dei documenti richiesti dalla Regione Veneto. Le riflessioni riportate in questo articolo sono state elaborate durante lo svolgimento di questa esperienza e si inseriscono all'interno di un più ampio programma di ricerca applicata sulle forme di governance territoriale sia in Italia sia all'estero. 
Struttura. L'articolo, dopo una breve riflessione introduttiva (1.) sulla necessità di ripensare le forme di governo dell'area vasta con il supporto della scala intercomunale, si focalizza sulla figura veneta delle Intese Programmatica d'Area e, in particolare, ricostruisce il percorso di riconoscimento dell'IPA Veronese (2.). Il nostro lavoro di ricerca è un'operazione di esplorazione critica di questa esperienza (3.), nelle cui conclusioni (4.) si discute dell'efficacia di questo tipo di figure nel governo dell'area vasta, fuori dalle cornici istituzionali previste nell'ordinamento italiano.

\section{La cooperazione intercomunale come forma di governo dell'area vasta}

Nel libro verde sulla coesione territoriale la Commissione Europea (2008; 3) riconosce che l'approccio integrato e la cooperazione multilivello sono fattori decisivi per superare le sfide territoriali correnti. Fra di esse, particolare rilievo sembrano assumere per gli ambiti non metropolitani, la promozione delle relazioni fra le città e il loro ambito di influenza, della gestione integrata dei sistemi legati a questi ambiti geografici allargati (patrimoni culturali, reti, mercato del lavoro...), della competitività regionale e del miglior sfruttamento del loro potenziale paesaggistico e ambientale. Questo documento, insieme ad altri ad esso legati, come la Leipzig Charter on Sustainable European Cities (2007) e la Territorial agenda of the European Union 2020 (Council of Ministers responsible for Spatial Planning and Territorial Development, 2011) hanno imposto all'attenzione dei governi nazionali la necessità di ripensare le modalità e le forme di governo del territorio, con l'obiettivo di gestire le dinamiche spaziali e pensare allo sviluppo, gettando ponti fra efficienza economica, coesione sociale ed equilibrio ecologico, ponendo il tema della sostenibilità al centro dell'elaborazione politica (Barca, 2009).

In un territorio eterogeneo e fortemente diversificato come quello europeo, quest'esigenza si è tradotta anche nella ricerca di forme di governance legate a scale di riferimento alternative a quelle istituzionali, che permettessero di comprendere e influenzare le dinamiche socio-economiche e spaziali in atto. Come ha riconosciuto Tubertini (2014), lo spostamento verso una scala sub-regionale di governance è servito a supportare lo sviluppo locale attraverso la sperimentazione di un modello diverso di ente intermedio, che dovrebbe favorire un governo del territorio più coerente $\mathrm{e}$ armonico, secondo una visione non più competitiva, ma di raccordo fra amministrazioni locali ed enti di coordinamento. In presenza di poli urbani rilevanti, il principio è stato quello di definire delle entità (come le métropoles in Francia...) che avrebbero dovuto superare la rigidità dei confini municipali e assumere un ruolo più incisivo rispetto a quello delle province. Per questo, in Italia alle Città Metropolitane (L. 56/2014) sono 
state affidate funzioni legate alla pianificazione strategica e generale, alla pianificazione urbanistica e allo sviluppo economico. In parallelo, come riportato in uno studio del Censis (2013), la realtà attuale del paese è lontana rispetto a quella descritta dalla mera suddivisione fra Province e Città metropolitane, specie in aree o regioni urbane, estese ma non incentrate su un grande capoluogo - come ad esempio Brescia, la Sicilia sud-orientale, Padova... - che sfuggono a questa logica bipartita. In questi contesti, la diffusione territoriale di materiali urbani come popolazioni, imprese, infrastrutture e servizi (Infussi, 2011) rimanda a un quadro spaziale più ricco e articolato, che richiede la definizione di una scala intermedia fra Comune e Regione in grado di rappresentare la 'giusta distanza' per leggere necessità e potenziale dello spazio, governare i fenomeni e dare una possibilità di sviluppo delle periferie e degli ambiti dinamici delle frange urbane e rurali.

Per questo, la configurazione istituzionale prevista all'interno della riforma, così come risultata allo stato attuale a valle anche del referendum costituzionale del 2016, è subito apparsa "sfuocata e giunta fuori tempo massimo" (Balducci e al., 2017c, p. 23), incapace di leggere le nuove condizioni territoriali in cui la distribuzione spaziale dei fenomeni urbani non corrisponde a quella delle istituzioni deputate a governarli. Le criticità riconosciute nell'assetto istituzionale attuale sono ancor più marcate negli ambiti non identificati come "città metropolitane" dove la dimensione transcalare e pervasiva delle "nuove questioni urbane" (Balducci e al., 2017d; Brenner e Schmid, 2015; Fedeli, 2011) non è considerata e rimane ancorata ai confini amministrativi dei comuni o delle regioni e dove le province sono enti di secondo livello senza effettiva capacità di incidere su questi temi.

L'ipotesi di lavoro iniziale entro cui si muove questo nostro contributo è che il governo dell'area vasta fuori da queste istituzioni sia possibile solo attraverso il ricorso a una serie di pratiche e/o figure che integrino i livelli istituzionali esistenti e rappresentino una discontinuità rispetto allo status quo, con l'obiettivo di rendere più efficiente ed efficace la gestione ordinaria del territorio e, al tempo stesso, di permettere la costruzione di interventi orientati al suo sviluppo.

Non si tratta di un approccio originale all'interno della pianificazione, tanto che gli Enti Locali sono già dotati di strumenti che permettono loro di cooperare con livelli diversi di coinvolgimento e durabilità nel tempo. Un quadro interpretativo dei tipi di intercomunalità praticati in Italia è stato elaborato da Fedele e Moini (2006).

Senza entrare nel merito di ognuna delle tipologie presentate, sembra importante ricordare, come ha fatto Vinci (2011), che fin dagli anni Novanta sono stati sperimentati con intensità diversi "strumenti di programmazione locale fondati su partnership tra soggetti pubblici portatori di competenze eterogenee e soggetti rappresentativi a vario titolo di interessi privati ma comunque territoriali" con risultati sullo sviluppo economico e sociale che 
sono stati valutati in termini prevalentemente negativi o, comunque, non esenti da rischi. Si tratta di esperienze attraverso le quali sono state istituite strutture e forme organizzative ad hoc (uffici speciali, agenzie locali...) per la gestione dei programmi e una moltiplicazione di centri di potere - e voci di spesa - nel governo delle politiche di sviluppo "che ha inevitabili effetti sull'efficacia dei processi decisionali e rende complessa la ricomposizione di risorse e visioni comuni per lo sviluppo territoriale" (Vinci, 2011).

Figura 1 - Tipi di intecomunalità (elaborazione autori su Fedele e Moini, 2006, p. 81)

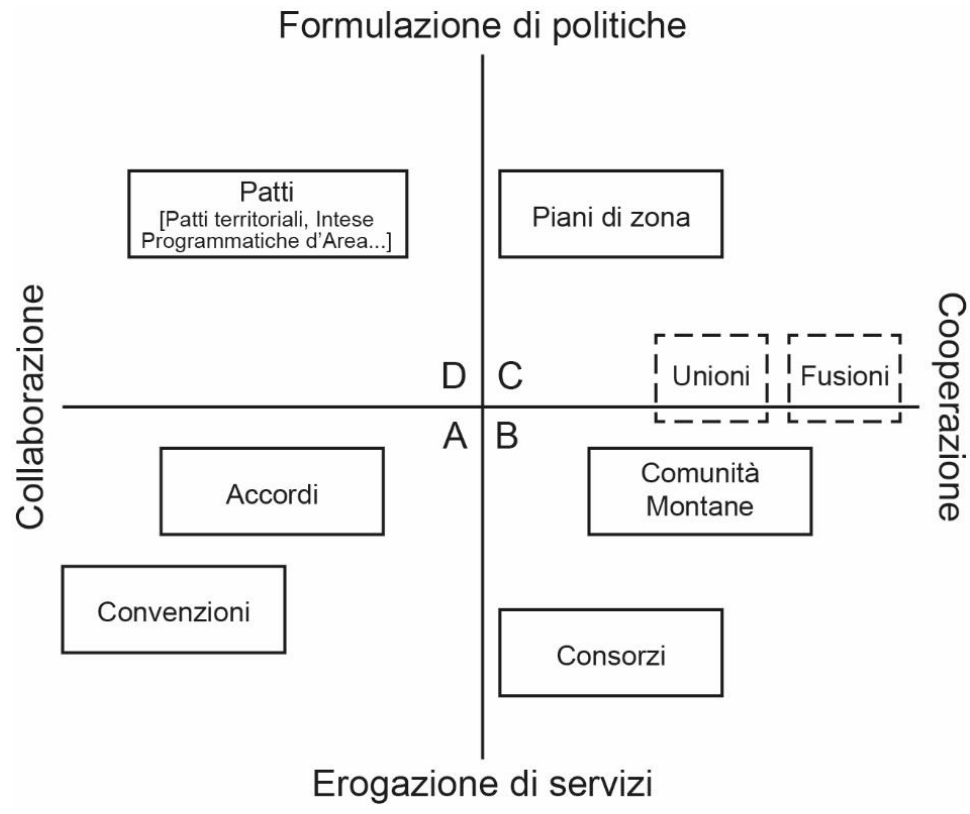

Lo schema della figura 1 invita a individuare tra gli strumenti che possiamo definire "dell'intercomunalità" quello più efficace per raggiungere uno specifico obiettivo. Se l'obiettivo per un territorio è migliorare l'efficienza dei servizi erogati dai Comuni, la convenzione può rappresentare lo strumento che è preferibile utilizzare poiché focalizza l'attenzione sul servizio. L'Unione di Comuni, invece, può rappresentare l'esito finale di un processo che abbia visto un gruppo di Comuni sperimentare per alcuni anni con successo e in accordo più convenzioni per la gestione dei servizi. Nella realtà stiamo invece assistendo a una forte spinta verso la creazione di Unioni tra Comuni ai quali manca una preliminare esperienza di gestione di più convenzioni, con il risultato che questo tipo di unioni che difettano di un solido training alla cooperazione non di rado si dissolvono dopo pochi anni di esperienza.

Se, invece, l'obiettivo di un territorio è costruire uno strumento per formulare/programmare politiche, quali in particolare quelle di sviluppo 
territoriale, allora la figura $1 \mathrm{ci}$ invita a puntare la nostra attenzione sui quadranti superiori ( $\mathrm{D}$ e $\mathrm{C}$ ) e in particolare sui patti territoriali, che sono esperienze di collaborazione nelle quali prevalgono forme di interazione scarsamente strutturate dal punto di vista formale e che trovano un significativo collante negli obiettivi di policy che si intendono raggiungere. Si tratta di strumenti articolati attorno ad un accordo di programma o più frequentemente a un protocollo d'intesa, con il quale si definisce la collaborazione tra diversi attori politico-istituzionali (Comuni e altri enti pubblici), soggetti rappresentanti di imprese e lavoratori, associazioni sociali, civili e di rappresentanza di interessi presenti in un determinato contesto locale per la formulazione di concreti programmi di sviluppo economico (Fedele e Moini, 2006, p. 85). Esempi di questo tipo di pratiche sono i patti territoriali e le varianti o evoluzioni di essi; strumenti per loro natura inclusivi, basati su un partenariato con composizioni e flessibilità diverse, che al tempo stesso riconoscono la centralità degli enti locali, e consentono quindi di concentrare l'attenzione su limiti e potenzialità di un "territorio" in quanto tale, ponendosi più di altri come strumenti che possono favorire i processi di sviluppo locale (Vino, 1998).

I patti territoriali sono una risorsa efficace per lavorare sulla governance territoriale che si integra meglio di altre con la strumentazione urbanistica esistente. Essi possono convivere parallelamente a forme stabili di cooperazione (come le unioni o le fusioni di Comuni) e, a differenza di altri tipi di intercomunalità, sono flessibili e adattabili dato che permettono la definizione di progetti specifici basati su collaborazioni orientate alle tematiche centrali dello sviluppo urbano sostenibile. Così come sottolineato da Vinci (2011), questi documenti impongono agli stati membri e, di conseguenza, alle loro autorità regionali di qualificare la propria azione strutturante dei territori privilegiando:

- l'incremento della capacità di indirizzo strategico dei territori regionali che coinvolge la definizione di obiettivi e campi d'azione, anche mutuati dal livello nazionale e comunitario. Questo scenario rappresenta la base per attivare $\mathrm{i}$ processi di negoziazione e cooperazione con le reti locali necessarie al montaggio delle politiche integrate per la rigenerazione economica, la qualificazione dei sistemi urbani, lo sviluppo sostenibile;

- la necessità di supportare la progettualità delle realtà locali attraverso processi di valutazione selettiva tali da permettere la costruzione di azioni solide e sostenibili da convogliare nei processi di sviluppo regionale e interregionale, catalizzando risorse e sforzi organizzativi su un numero più limitato di obiettivi e territori.

Si tratta di richieste che spesso rimangono inevase anche a causa degli attuali assetti istituzionali e rispetto alle quali è possibile cercare forme innovative di azione, ad una scala che trascenda i confini amministrativi attuali e che lavori con riferimento all'area vasta. Per confermare questa ipotesi, si è scelto di presentare un caso di studio nelle pagine successive, che 
permette di mettere in luce caratteri innovativi, potenzialità e rischi connessi a queste pratiche. In questo senso, il percorso di riconoscimento dell'Intesa Programmatica d'Area veronese ha rappresentato un laboratorio di sperimentazione per le politiche pubbliche del territorio (Vino, 2012) e per le interazioni fra attori diversi alla scala inter-comunale.

\section{L'IPA Veronese}

Fin dagli anni Ottanta la Regione Veneto ha predisposto strumenti di pianificazione e programmazione sovracomunale che presentano configurazioni e sistemi operativi diversi, articolati in funzione degli obiettivi da perseguire. La pianificazione dello sviluppo economico di area vasta o semplicemente di aree sovracomunali si è sviluppata grazie allo strumento dell'Intesa Programmatica d'Area (IPA). Questa figura, versione evoluta dei patti territoriali, è stata introdotta nell'ordinamento regionale veneto nel 2001 con l'art. 25 della L.R. 35 allo scopo di attivare "metodi di co-decisione con cui formulare proposte politiche con l'obiettivo di incidere non solo sulla programmazione regionale ma anche su quella degli stessi Comuni coinvolti, vincolando su base volontaria le politiche e gli strumenti di programmazione dei soggetti agli obiettivi e alle strategie comuni" ${ }^{3}$. Con la successiva Deliberazione della Giunta Regionale 2796/2006 sono state precisate le modalità per l'istituzione delle IPA mediante un processo bottom-up e il successivo riconoscimento della Regione, passando attraverso la costituzione di partenariati composti da soggetti pubblici e privati che, in forma associata, operano per contribuire allo sviluppo del proprio territorio attraverso la definizione di accordi e la formulazione di proposte finalizzate allo sviluppo economico-sociale di ambiti sub-regionali.

Questi principi hanno attuato e, di fatto, anticipato le istanze proposte dalla Leipzig Charter dove si spingevano gli stati membri dell'UE a promuovere le strutture più opportune per garantire uno sviluppo urbano integrato e ad implementare forme di governance efficaci per raggiungere una organizzazione territoriale equilibrata e policentrica.

La flessibilità nella costruzione del partenariato, la possibilità di orientare la sua azione verso obiettivi specifici e la capacità di risposta alle istanze della programmazione europea mostrano la natura evidentemente diversa delle IPA rispetto agli enti e strumenti a livello intermedio - in primo luogo Province e Unioni di Comuni - e si inseriscono in un sistema più ampio di figure e/o soggetti istituzionali creati con finalità, procedure e strumenti specifici.

Dopo una prima stagione (2001-2004) nella quale i patti territoriali generalisti (18 in Veneto) avevano maturato "ope legis" le condizioni

\footnotetext{
${ }^{3}$ Regione Veneto, DGR n. 3517 del 06 novembre 2007. Programmazione decentrata Riconoscimento Intese Programmatiche d'Area (IPA). (Art. 25 1.r. 35/2001; DGR 2796/2006).
} 
necessarie per essere considerati tali, la Regione Veneto ha riconosciuto altre 3 IPA nel 2005-2007, 3 nel 2008, 2 nel 2009, 2 nel 2010 e nel 2012 fino a un totale di 24 IPA attualmente attive sul territorio regionale ${ }^{4}$.

Nel biennio 2013-2014, in un ambito della Provincia di Verona (la "fascia centrale" attraversata dall'autostrada A4) dove non era stata attivata un'Intesa Programmatica d'Area, la volontà di confronto espressa da amministratori e tecnici ha rappresentato una spinta a riflettere sulle opportunità legate a questa forma di cooperazione. Rispetto alle altre esperienze pattizie, l'IPA permette di mettere a sistema progetti e risorse e di disporre di uno strumento "leggero" e al tempo stesso efficace con il quale affrontare i temi dello sviluppo urbano sostenibile dell'area. Inoltre, politici e tecnici locali avevano espresso la necessità di disporre di un riferimento di area vasta in grado di orientare le politiche e le azioni degli enti locali nel campo dello sviluppo del territorio. Al tempo stesso, segnalavano il bisogno di dotarsi di strumenti interpretativi, politici e finanziari per affrontare le sfide cui è sottoposta l'area vasta di riferimento.

Tra le esigenze manifestate da più soggetti, segnaliamo anche il bisogno di innovare i processi di governance attraverso cui supportare i Comuni e i partner aderenti nella definizione degli obiettivi strategici, delle politiche condivise e delle azioni da attuare sul territorio alla scala vasta.

L'ambito interessato all'IPA Veronese coincide con il territorio di 14 Comuni (Bussolengo, Buttapietra, Castel d'Azzano, Castelnuovo del Garda, Lazise, Pastrengo, Pescantina, San Martino Buon Albergo, San Pietro in Cariano, Sommacampagna, Sona, Valeggio sul Mincio, Verona, Zevio), occupa una superficie di $632,23 \mathrm{~km}^{2}(3,5 \%$ sul totale della superficie della Regione Veneto) dove sono localizzati 419.675 abitanti (8,5\% della sua popolazione).

La scelta di lavorare su questo specifico spazio è legata a criteri di natura spaziale e politica. Il territorio in esame è caratterizzato da alcuni fattori insediativi peculiari che lo rendono uno spazio dotato di un'identità specifica all'interno della fascia centrale della provincia di Verona. Tra questi fattori segnaliamo: (i.) un'alta densità abitativa ${ }^{5} \mathrm{e}$ di unità locali d'impresa ${ }^{6}$, (ii.) una marcata vocazione legata ai servizi e al turismo e (iii.) una dimensione

\footnotetext{
${ }^{4}$ Il totale non corrisponde alla somma delle IPA riconosciute perché alcune di esse hanno subito processi di accorpamento, trasformazione e/o scioglimento.

${ }^{5}$ Nell'ambito dell'IPA Veronese la densità abitativa è pari a $664,8 \mathrm{ab} . / \mathrm{km}^{2}$, valore più che doppio rispetto alla media regionale $\left(268 \mathrm{ab} . / \mathrm{km}^{2}\right)$, a quella provinciale $\left(293 \mathrm{ab} . / \mathrm{km}^{2}\right) \mathrm{e}$ a quello della fascia centrale della Provincia di Verona $\left(432 \mathrm{ab} . / \mathrm{km}^{2}\right)$, ambito che rappresenta il contesto territoriale dell'IPA stessa.

${ }^{6}$ Il dato di 52.240 unità locali presenti nel registro delle imprese per i comuni appartenenti all'IPA (Dati: Camera di Commercio, 2014) ben rappresenta l'eccezionale densità di imprese all'interno dell'IPA $\left(82,66 \mathrm{UL} / \mathrm{km}^{2}\right)$, tanto più se si considera questo dato in relazione alla media italiana $\left(23,9 \mathrm{UL} / \mathrm{km}^{2}\right)$, quella della Regione Veneto $\left(32,28 \mathrm{UL} / \mathrm{km}^{2}\right)$, quella della Provincia di Verona $\left(37,08 \mathrm{UL} / \mathrm{km}^{2}\right)$.
} 
produttiva legata all'agricoltura ${ }^{7}$, alla manifattura e all'agro-alimentare che contribuisce ad attivare un paesaggio urbano-rurale complesso.

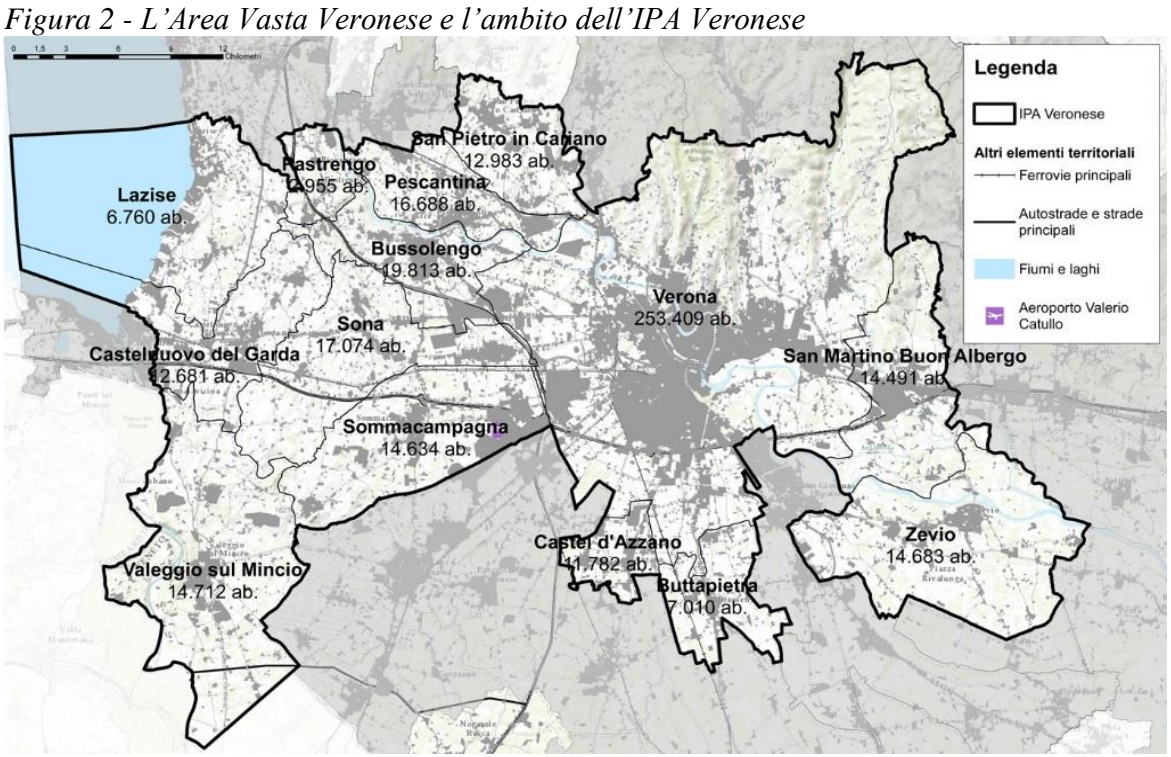

Questa identità specifica, assieme alla volontà politica espressa trasversalmente dagli amministratori di confrontarsi con le esternalità legate a dinamiche e fattori che influenzano lo sviluppo attuale e futuro di quest'area, fra cui il turismo, le infrastrutture AV/AC dei corridoi europei, la presenza di uno stock immobiliare - anche pubblico - da recuperare e/o riattivare... sono divenute le ragioni per provare a costituire un tavolo di partenariato configurato come IPA.

È stato pertanto condotto un lavoro di analisi territoriale mediante il quale è stato possibile esplorare la condizione di un'area nella quale la morfologia, le condizioni economiche e sociali, le evoluzioni storico-culturali e politiche, hanno dato luogo a uno spazio articolato. Quest'ultimo è ricco di potenzialità legate alla solidità dei sistemi produttivi ed economici presenti, ma anche di contraddizioni, dove la mancanza di coordinamento e l'assenza di una visione territoriale condivisa hanno generato squilibri e differenze sia spaziali che economico-sociali. Dal punto di vista metodologico, si è trattato di raccontare attraverso la produzione di uno specifico "spatial knowledge"

${ }^{7}$ I dati raccolti dalle Camere di commercio (2014) permettono di osservare che rispetto alla media regionale, per cui solo il $4,1 \%$ degli addetti era impiegato nel settore dell'agricoltura, nell'ambito dell'IPA il valore è quasi doppio $(8,01 \%)$ e superiore anche alla media registrata considerando tutti i comuni della fascia centrale della provincia di Verona $(5,1 \%)$. 
(Paris, 2017) questa condizione di urbanizzazione complementare, frammentaria e allargata (Fregolent e Vettoretto, 2017).

In questo tipo di contesto, la figura dell'IPA permette di definire alcune strategie di azione che rispondono alle priorità definite nell'agenda territoriale europea ${ }^{8}$ e che non sarebbero perseguibili attraverso l'azione delle istituzioni ora operanti nell'area. In particolare, ci si riferisce alla necessità di promuovere sviluppo territoriale attraverso la costruzione di relazioni integrate e multilivello fra città e spazi rurali, e alla capacità di adattare le istituzioni alle realtà locali. Per questo, la scelta di lavorare con uno spazio di dimensioni relativamente contenute rispetto al totale dei 52 Comuni della fascia centrale della provincia di Verona, rappresenta un'opportunità che permette di mettere alla prova i protocolli definiti per il funzionamento dell'IPA, di valutarne l'efficacia e misurarne gli impatti, con la possibilità di estendere successivamente l'area di azione. Inoltre la presenza di un numero limitato di attori che, pur legati a diversi orientamenti e approcci politici e tecnici, mostrano una forte motivazione a collaborare, consente di testare i meccanismi di rappresentanza disperdendo la minor quantità possibile di energie.

In parallelo, la figura dell'IPA permette di lavorare sugli aspetti di governance, testando meccanismi di coordinamento territoriale, di democrazia interna e rappresentatività, di interazione collaborativa fra partner privati e attori pubblici e l'efficacia di potenziali processi di scaling up dei protocolli ${ }^{9}$ implementati (Lingua, 2017). Per questo, la presenza di un limitato numero di attori accresce in termini relativi il peso demografico e politico della città di Verona e rispetto a ciò, la scelta iniziale non preclude la possibilità di un allargamento. Infatti, la futura inclusione di nuovi Comuni e attori privati nel partenariato potrà essere valutata nel medio periodo, anche in funzione dell'impatto di queste scelte sugli obiettivi generali, sulla capacità di produrre scenari condivisi e sull'interesse manifestato dai candidati interessati a entrare nell'IPA Veronese.

Nei paragrafi successivi la ricostruzione del percorso finora effettuato nell'ambito del riconoscimento dell'IPA e degli obiettivi preposti serve ad approfondire aspetti metodologici e procedurali del processo ma, allo stesso tempo, a focalizzare quegli elementi che possono essere considerati come innovativi per il governo dell'area vasta.

\subsection{Il processo di riconoscimento per fasi}

\footnotetext{
${ }^{8}$ Cfr. Territorial agenda of the European Union 2020, III Territorial Priorities for the Development of the European Union.

${ }^{9}$ Cfr. Territorial agenda of the European Union 2020, IV. Making EU territorial cohesion a reality - "The governance and implementation mechanisms".
} 
Nei documenti prodotti per costituire e attivare l'IPA Veronese, è descritto l'iter di definizione dell'ambito territoriale interessato e dei suoi obiettivi come un processo di "riconoscimento" che deve essere effettuato a due livelli.

Il primo livello di riconoscimento interessa gli abitanti, gli amministratori e i tecnici che vivono e si muovono in quest'ambito e che faticano a pensare ai Comuni dell'area come a parti di un sistema territoriale integrato. Questa difficoltà, dal punto di vista delle istituzioni e della componente politica del partenariato, è dovuta a una tradizionale ritrosia di alcuni componenti a collaborare in un sistema in cui la città di Verona assume un ruolo di capofila, tanto che in altri due tentativi precedenti (2004 e 2012) non si erano create le condizioni per la costituzione di un'Intesa Programmatica d'Area nello stesso ambito. Dal punto di vista degli abitanti e delle imprese, è in atto un lento ma costante processo di integrazione fra pratiche e processi locali, che stanno portando a una progressiva collaborazione degli attori su alcuni temi quali l'esplorazione rispetto a una possibile istituzioni di un Unione di Comuni in una parte dell'ambito, la condivisione di esperienze tra più Comuni sui servizi di informazione turistica.

Il secondo livello di riconoscimento riguarda gli organi regionali, che devono riconoscere la costituzione di quest'entità validandone atti costitutivi e documenti programmatici. In questo caso si tratta di un percorso segnato da almeno tre momenti distinti, marcati da un progressivo avvicinamento all'ambito spaziale di lavoro, degli obiettivi e dei progetti previsti per l'IPA Veronese.

La prima fase, corrispondente alla seconda metà del 2014, è legata all'avvio delle operazioni. In questo periodo, le frequenti relazioni sia tecniche, sia politiche avviate da alcuni Comuni dell'area, in particolare quelli di Verona e Valeggio sul Mincio, hanno reso evidente il potenziale legato alla costruzione sia di un tavolo di confronto e discussione rispetto ad alcuni temi settoriali (infrastrutture, turismo, agricoltura) sia di uno spazio di costruzione di una cornice strategica entro cui inquadrare le singole azioni.

Per questa ragione, tecnici e politici hanno individuato l'IPA come lo strumento collaborativo adatto a rispondere alle esigenze di un territorio ricco di risorse, patrimonio e capitale sociale ma poco abituato a cooperare sia nell'ambito delle pratiche amministrative, sia per quanto riguarda la prefigurazione di scenari di opportunità. Questa prima fase ha portato nel gennaio 2015 alla costituzione dell'IPA in forza di un partenariato cui partecipano i rappresentanti dei 14 Comuni interessati, della Camera di Commercio di Verona, del Dipartimento di Informatica dell'Università degli Studi di Verona e di alcune Associazioni di rappresentanza delle imprese del territorio. Questo impegno si è concretizzato nella presentazione alla Regione del Documento programmatico d'area intitolato "Analisi del territorio dell'Area Vasta Veronese. Quattro assi strategici, un unico campo 
d'azione" e del Protocollo d'intesa ${ }^{10}$ che regola il partenariato, definito "tavolo di concertazione" 11 , che i partner hanno approvato, corredato di regolamento interno. In quest'ultimo, è riconosciuto il ruolo di pivot e coordinatore dell'IPA a una segreteria tecnica, composta da funzionari in servizio e da alcuni esperti chiamati a supportarne i lavori.

La seconda fase, apertasi in seguito alle osservazioni pervenute dalla Regione Veneto, riguarda la costruzione di una strategia di sviluppo sostenibile per l'area dell'IPA. In questo caso, anche seguendo le indicazioni provenienti dalla Sezione Affari generali e FAS-FSC della Regione, si è reso necessario concretizzare i principi indicati nel Documento programmatico d'area, arrivando alla definizione di una visione comune per il territorio. Questo ha portato alla successiva individuazione di un sistema di obiettivi settoriali, ma interconnessi, da raggiungere attraverso azioni puntuali.

Per questo si è resa necessaria la comprensione della realtà spaziale contingente, delle tendenze economiche e delle declinazioni locali di alcuni orientamenti provenienti da una pluralità di ambienti, culture e contesti, quali la politica locale e regionale, la finanza, il contesto normativo. In questo modo è stato possibile leggere in modo consapevole gli effetti di alcuni processi contemporanei in atto nell'area (quali la metropolizzazione del territorio, la globalizzazione e de-localizzazione di attività economiche, lo spopolamento di alcune aree e la densificazione di altre...) e provare a governarne le esternalità. Allo stesso tempo, è necessario portare all'interno della strategia per l'IPA Veronese le necessità e le istanze locali che pur partendo "dal basso" e dal quotidiano possono divenire la chiave di alcuni asset futuri di questo territorio, come la capacità attrattiva, lo sviluppo socioeconomico sostenibile e la qualità della vita. La presentazione del documento "Verso una strategia di sviluppo economico sostenibile dell'Area Vasta Veronese" dell'ottobre 2015 manifesta un passo importante per l'IPA, poiché è un importante frutto di questo percorso.

La riflessione prodotta dalla Regione Veneto dopo la ricezione di questo documento ha aperto la terza fase del lavoro, nella quale è stata presentata una nuova versione del Documento Programmatico d'Area in cui è stato approfondito il lavoro di conoscenza sul territorio dei 14 Comuni che compongono l'IPA Veronese, compiendo uno sforzo significativo nella raccolta e costruzione di un catalogo con un centinaio di programmi/progetti in corso, un'analisi orientata delle necessità comuni e una valutazione delle opportunità presenti. Ciò ha permesso di puntare con maggior forza

\footnotetext{
${ }^{10}$ Questi tre documenti sono considerati elementi necessari alla costituzione di una IPA ai sensi della DGR 2796 del 12 settembre 2006 della Regione Veneto.

${ }^{11}$ Il tavolo di concertazione, così come espresso nel Regolamento interno sottoscritto dagli aderenti e presieduto dal Sindaco pro-tempore (o da un suo delegato) del Comune capofila di Verona, è composto dai rappresentanti dei soggetti pubblici e privati (in forma associata) che decidono di sottoscrivere il Protocollo di intesa per la costituzione dell'IPA Veronese.
} 
sull'innovazione nei processi progettuali attraverso la creazione di una "centrale dei progetti" quale carattere distintivo dell'Intesa.

\subsection{La centrale dei progetti dell'IPA Veronese come protocollo innovativo}

L'obiettivo dell'IPA Veronese non è la costruzione di una lista degli interventi auspicati dai singoli membri, con il rischio che la concertazione e la co-progettazione siano relegate alla fase di esercizio più che orientare le scelte politiche e tecniche dei partner nel medio periodo. Il rischio di un'operazione del genere è la trasformazione dell'Intesa in un ulteriore livello amministrativo di raccordo: ciò la svuoterebbe di quella agile carica dinamizzatrice che ne caratterizza lo spirito. L'IPA Veronese, nelle intenzioni dei suoi promotori, dovrebbe, al contrario, rappresentare la risposta ad alcune delle necessità del territorio che si manifestano in una domanda di supporto dal punto di vista strategico e, per alcuni aspetti, gestionale che i Comuni aderenti - ma anche le Associazioni di rappresentanza delle imprese - si trovano a dover affrontare nel quotidiano svolgimento delle loro funzioni. È anche questo il motivo per cui il carattere distintivo dell'IPA Veronese è che non si costituisce attorno ad un progetto o un fine specifico ma con riferimento a un territorio che individua caratteri, problematiche e potenzialità comuni e attorno alle quali vuole lavorare con protocolli strutturati per l'implementazione delle progettualità locali. Queste progettualità dovrebbero essere definite nel tempo e con continuità tra gli attori locali in relazione alle risorse finanziarie e umane, ai bisogni e alle opportunità all'interno del tavolo di concertazione.

Segue questo metodo anche la creazione di un dispositivo all'interno dell'Intesa - la centrale dei progetti - che dovrebbe servire a raccogliere e implementare i progetti proposti dai singoli partner attraverso la condivisione di risorse sia tecniche sia finanziarie, e che permetta di costruire azioni solide ed efficaci come risposta a bisogni e necessità condivise.

Figura 3 - Lo schema di funzionamento della centrale dei progetti dell'IPA Veronese 


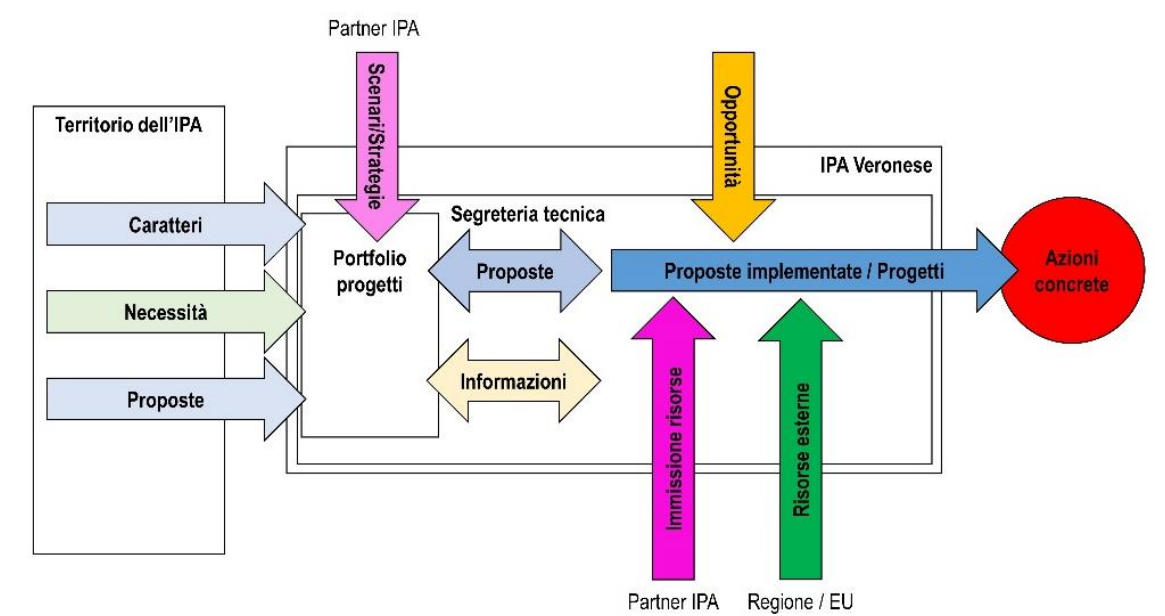

Il portfolio dei progetti dell'Intesa, costantemente aggiornato grazie al lavoro della segreteria tecnica, permette di costruire un insieme di azioni, marcate da gradi diversi di sviluppo e attuabilità, atte a materializzare gli scenari e le strategie definite all'interno del tavolo di partenariato. In questo modo la cooperazione fra i partner si articola attorno a proposte concrete cui possono aderire in modo volontario e in funzione delle singole necessità. In particolare, evidenziamo che per ciascun progetto promosso dall'Intesa si hanno delle aggregazioni diverse in relazione alle tematiche, alle sub-aree, agli interessi dei componenti stessi dell'IPA. Si tratta, in questo caso, di una scelta consapevole, che punta a privilegiare la costituzione di gruppi coesi e motivati all'interno dell'IPA, evitando di imporre costi organizzativi, economici e di coinvolgimento ad attori non interessati direttamente ad ogni singolo progetto. Le proposte dovrebbero poi essere perfezionate e raggiungere un grado di definizione sempre maggiore (anche dal punto di vista del livello di progettazione e del finanziamento) e tradursi in azioni concrete, seguendo un processo incrementale che può trarre vantaggio dalle opportunità offerte dalla programmazione regionale ma che articola gli interventi all'interno di una propria specifica agenda strategica.

Questa impostazione organizzativa dell'IPA Veronese ha delle ricadute dirette anche sulla definizione delle risorse (finanziarie e organizzative) necessarie per l'attuazione e la gestione a livello locale dei programmi della stessa Intesa e sulle modalità di cooperazione tra i soggetti che partecipano al partenariato. Il vero obiettivo è quello di fornire uno spazio di confronto e costruzione di scenari strategici e orizzonti di senso entro cui orientare le politiche, i programmi e le azioni dei singoli componenti. La sfida è riuscire a mettere a sistema esigenze e potenzialità locali e di costruire un meccanismo che garantisca in egual maniera supporto e libertà all'azione dei singoli Comuni, che orienti e cerchi di indirizzare le azioni degli attori privati nel territorio, ma che riesca anche a riunire gli sforzi individuali per raggiungere obiettivi comuni nel campo dell'economia, dell'ambiente e della 
governance, con influenze dirette sulla qualità della vita degli abitanti dell'area.

\section{La cooperazione intercomunale come laboratorio: temi emergenti, apprendimenti e rischi}

La scala intercomunale è, come afferma un recente rapporto di ricerca del Censis (2013), la dimensione dove si esprimono le dinamiche più interessanti per quanto concerne i processi lavorativi, la mobilità, lo studio, la produzione, l'attrattività turistica.

Figura 4 - Presenze turistiche nell'ambito dell'IPA Veronese (Dati: Camera di commercio Verona, 2014)

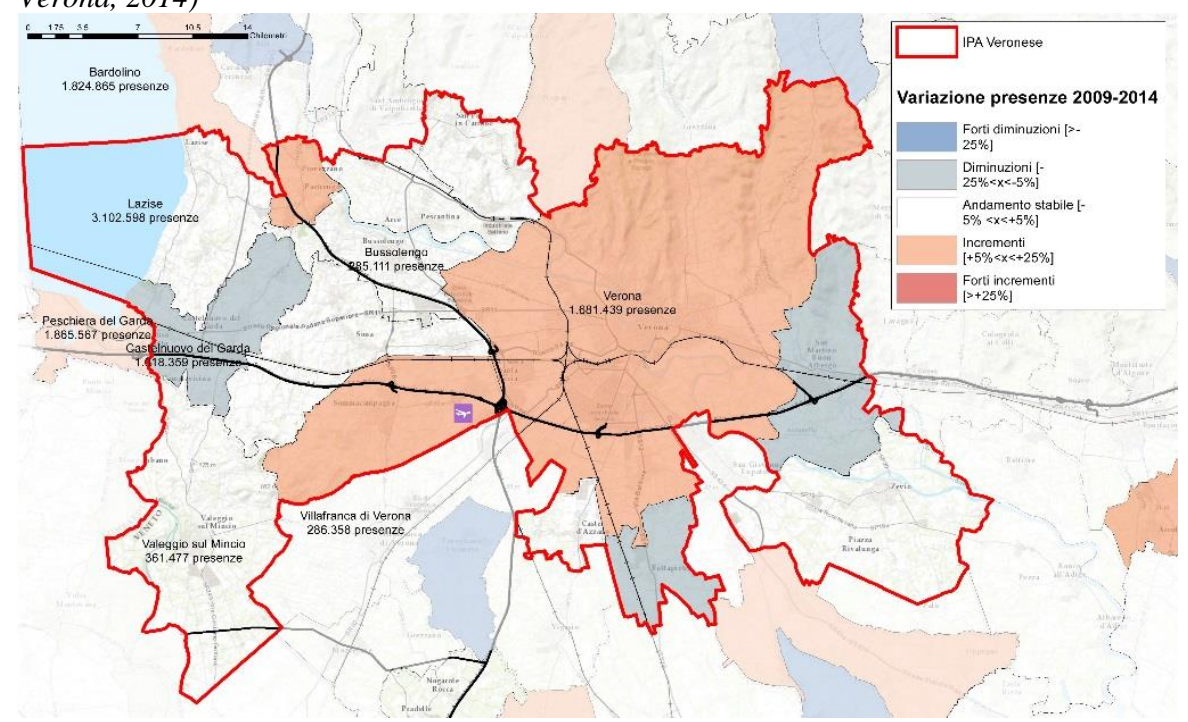

Gli ambiti infra-regionali da questa definiti contengono le realtà reticolari dei processi produttivi, delle pratiche dell'abitare e delle filiere dei consumi di beni, servizi ed esperienze che caratterizzano la società contemporanea. Esse sono, dunque, il livello istituzionale più adeguato per interpretare le complesse dinamiche e organizzare gli esiti spaziali di queste realtà. A questa scala, strategie e immagini per il territorio devono tener conto delle esigenze e dalle potenzialità locali, così come dei diversi ruoli che i singoli stakeholders coinvolti possono apportare. I tentativi recenti di attivazione di forme di intercomunalità aperti e coerenti con le istanze europee, implementati sia a livello statale che regionale, tendono a configurarsi come processi complessi e la loro osservazione permette di indagare questa realtà come laboratorio di forme innovative di governance. 
Si è detto che il duplice percorso di riconoscimento dell'Intesa Programmatica d'Area Veronese - da parte sia della Regione Veneto sia dei suoi abitanti - è ancora in corso. Al tempo stesso, quest'esperienza ha permesso l'acquisizione di alcuni apprendimenti e, guardando al percorso compiuto finora, si può riflettere sulle opportunità e i rischi legati alla cooperazione inter-comunale al di fuori delle città metropolitane, assumendo una prospettiva critica rispetto ad alcune tendenze emergenti e moduli procedurali.

In particolare, segnaliamo:

- il superamento delle contingenze amministrative nelle necessità dei Comuni e degli Enti Locali anche in ambiti dove la gerarchia urbana non è descrivibile solo attraverso la logica centro-periferia. Gli attori pubblici coinvolti nel governo locale richiedono maggiori risorse e/o maggiori libertà nel loro utilizzo, ma non solo. Spesso attendono che gli enti di scala sovraordinata forniscano un set di proposte, di orientamenti, di linee guida che diventino una road map per le strategie e le azioni locali - che poi svilupperanno autonomamente - e che oggi mancano. In questo senso le IPA e in generale le figure di governo dell'area vasta dovrebbero servire ad evidenziare programmi e risorse, che possono divenire la base per immaginari e scenari, ma anche per la creazione dei sistemi e delle reti necessari a rendere concrete queste visioni. Al tempo stesso, l'attenzione per gli aspetti strategici e di orientamento non deve però allontanare il focus dalla dimensione delle realtà del territorio e/o dalla costruzione di azioni specifiche, onde evitare il rischio della "concertazione vuota" (CNEL, 1997) attraverso cui le interazioni fra Enti Locali e attori privati non producono proposte concrete e risultano incapaci di attirare finanziamenti;

- la necessità di un coinvolgimento "modulare" degli Enti Locali e degli stakeholders nei processi di collaborazione. I soggetti che fanno parte del governo dell'area vasta mediante patti territoriali chiedono di essere coinvolti in modo flessibile e volontario nelle azioni programmate, con la possibilità di discutere e decidere di partecipare ad azioni e progetti specifici, anche in funzione di momenti e opportunità contingenti. Si tratta di un approccio in cui le iniziative possono essere promosse dal basso, estendendo ad altri quanto elaborato da uno specifico Comune, ma anche dall'alto, grazie alla possibilità di declinare sul territorio le istanze regionali, nazionali o comunitarie. Viene così valorizzata la volontarietà dei singoli membri nella partecipazione, come ad esempio nella costruzione di programmi e politiche attive senza per questo essere costretti ad entrare in azioni o interventi che non suscitano l'interesse di un singolo partner o esulano, magari solo temporaneamente, dalle loro possibilità. Nel caso di studio presentato, questo è possibile grazie al doppio canale su cui lavora l'Intesa, che se da un lato si occupa di fornire strumenti e progettualità a breve o medio termine, dall'altro costruisce scenari e visioni su cui le singole azioni trovano coerenza e che costituiscono l'intelaiatura su cui le 
amministrazioni pubbliche locali possono lavorare con affondi e interventi. Al tempo stesso il ruolo di raccordo dell'agire in partenariato è utile per evitare il rischio di conflitti istituzionali originati da sovrapposizioni di competenze fra enti di natura diversa e/o dal rischio di esclusione connaturato a queste figure di cooperazione, che potrebbero originare dispersione di risorse e inefficienze nello sviluppo di azioni specifiche (cfr. DIPE, 2015). In sintesi, questo schema di governance dell'area vasta definisce una modalità flessibile di partenariato tra gli attori, che risulta essere particolarmente efficace nel regolare i rapporti tra pubblico e privato;

- l'interesse verso tematiche specifiche mostrato da un panel di attori anche istituzionalmente diversi (enti locali, rappresentanze di categoria, imprese). Nel caso dell'IPA Veronese, si nota come i temi del turismo, dell'agricoltura, della gestione dei rifiuti e quella del patrimonio edilizio esistente raccolgono diversi tipi di progettualità (ma anche di domande e di pressioni) che solo una visione sovraordinata può cercare di mettere a sistema e di strutturare entro un'idea di territorio coerente. Il rischio, in questo caso, è legato sia alla capacità di definire ambiti territoriali discreti attraverso chiavi interpretative legate a fattori diversi (geografici, socioeconomici...) o a processi selettivi, sia alla capacità di individuare ambiti d'azione trasversali e specifici, che possano essere influenzati da politiche e azioni sviluppate alla scala intercomunale. Per questo le letture territoriali proposte nei documenti legati al riconoscimento dell'Intesa, definiscono un ambito concreto (il territorio dei 14 Comuni aderenti) e uno scenario più ampio (52 Comuni della fascia centrale della Provincia di Verona) dove alcune delle dinamiche analizzate trovano una definizione più compiuta e diffusa. La definizione dello spazio di azione dipende quindi da fattori sia territoriali sia politici (opportunità, volontà dei singoli, apertura ai processi collaborativi). Entrambi questi aspetti devono essere esplorati e rappresentano un'incognita per l'efficacia e la possibile implementazione dei processi cooperativi;

- $i$ tempi di riconoscimento delle diverse forme di cooperazione intercomunale da parte delle istituzioni e da parte dei loro abitanti sono diversi rispetto a quelli, più lenti, necessari alla loro implementazione da parte dei partner. L'esperienza dell'IPA Veronese mostra come un percorso di questo tipo si muova almeno con due diverse velocità, che sono legate alle dinamiche politiche e amministrative da un lato e alla domanda di strumenti e azioni dall'altra. Per questo, diversi segnali mostrano la necessità - e la potenziale efficacia - della presenza di un tavolo di orientamento per i progetti e gli interventi dentro e fuori dall'area dell'IPA Veronese. Questo aspetto dimostra come la realtà del territorio e delle sue necessità anticipi, e in parte superi, le esigenze istituzionali, imponendo una riflessione sulla necessità di adattare alcune figure regionali già esistenti a nuove istanze degli Enti Locali. In parallelo, è necessario riflettere sui 
rischi che questi processi volontari nati per iniziative "dal basso" che aggregano soggetti diversi corrono nella generazione di partenariati "monchi" (CNEL, 1997: 12), marcati dall'assenza di uno o più soggetti rilevanti per un territorio a causa di circostanze contingenti o di opportunità. Questa possibilità ricorre anche nel caso di studio, con l'attuale assenza del Comune di Villafranca di Verona all'IPA Veronese, che ha deciso di non aderire al tavolo di concertazione nella sua prima fase di sviluppo. La mancanza di un referente così importante per il sistema logistico e infrastrutturale limita l'efficacia e la portata delle riflessioni sul sistema dell'accessibilità e le sfide poste dal futuro completamento dei due corridoi AV/AC all'ambito territoriale dell'IPA.

Tracciato questo primo quadro, si può dire che le forme di intercomunalità fuori dalle città metropolitane e in particolare le forme di governo mediante patti territoriali si configurano come tavoli per la discussione fra gli attori che in questo modo superano gli approcci e i tentativi che spesso caratterizzano l'operato degli Enti Locali, che disperdono energie e risorse finanziarie, umane e tecniche con progetti legati ad attività contingenti (abbattimento dei costi) o estemporanee ("inseguimento" di bandi regionali ed europei). È importante ricordare che nel caso dei patti si tratta di forme di governance di tipo "federativo" e di uno spazio di mediazione che si rivolge a un territorio complesso e variegato, nel quale si coordinano idee e interventi, pensati e promossi in modo condiviso e che "trovano spazio" attraverso geometrie variabili. La partnership composta da soggetti pubblici e privati, pensata come nel caso approfondito in questo articolo, si configura come soggetto pivot endogeno con un ruolo di servizio sia verso le comunità locali sia verso gli altri stakeholders. In questo senso i vari attori possono recitare la parte di soggetti trainanti per specifici progetti e ricoprire, quindi, il ruolo di animatori territoriali e agenti di sviluppo e allo stesso tempo trarre vantaggio dalla loro conoscenza del luogo e dalla loro appartenenza ad una specifica policy community.

\section{Conclusioni}

Questo articolo nasce dall'esigenza di riflettere nell'ambito degli studi regionali in qualità di pianificatori territoriali e ricercatori, sulla capacità delle forme di intercomunalità attualmente più diffuse di supportare la costruzione di quadri strategici e di scenario degli enti pubblici locali. Sulla scorta di quanto proposto da Balducci e Bertolini (2007), abbiamo scelto di farlo riflettendo "con le pratiche", presentando il caso del percorso di riconoscimento dell'IPA Veronese, in cui siamo stati chiamati a collaborare in qualità di esperti. I paragrafi precedenti contengono un confronto dal quale emerge la possibilità che su tutto il territorio e non soltanto nelle aree urbane di rango elevato sia presente un livello intermedio tra Provincia e Comune 
nel quale gli attori (istituzionali e non) possano interagire e far scaturire nuove esigenze e nuove opportunità di crescita e di sviluppo per i cittadini.

Più nello specifico, dalle nostre ricerche sul campo è emerso che a un livello intermedio di intercomunalità - che nel nostro caso è l'area centrale della provincia di Verona - possono essere attivati due strumenti:

a) la creazione e il funzionamento di un patto territoriale (nel nostro caso l'IPA Veronese) di area vasta finalizzato al raggiungimento di specifici e definiti obiettivi condivisi di sviluppo economico del territorio, senza che ciò sia accompagnato da un parallelo assetto istituzionale o da strutture amministrative permanenti;

b) l'attivazione di convenzioni per la gestione congiunta di funzioni amministrative tra più Comuni in alcune sub-aree omogenee dell'area vasta.

Questi due strumenti possono operare contemporaneamente, poiché il patto territoriale è focalizzato sulle funzioni dei quadranti superiori della figura 1 , mentre le convenzioni sono focalizzate sulle funzioni dei quadranti inferiori, vale a dire sull'erogazione di servizi.

Il patto territoriale, rappresentato nel nostro caso dall'IPA Veronese, si configura, quindi, come un tavolo di lavoro volontario sui temi dello sviluppo economico sostenibile per un'area vasta di medie dimensioni, all'interno della quale è auspicabile che sorgano almeno quattro o cinque sub-aree in corrispondenza delle quali i rispettivi Comuni intraprendano un processo che - in funzione delle necessità e delle potenzialità locali e delle occasioni puntuali - porti alla costruzione di forme di collaborazione sempre più strette, rappresentate prevalentemente da convenzioni per la gestione di servizi. I temi delle modalità di cooperazione, dell'integrazione fra gli aspetti pratico-amministrativi e quelli strategici, così come il monitoraggio dell'efficacia delle azioni intraprese, rimangono questioni aperte, così come sono da approfondire per l'ambito italiano le modalità di coinvolgimento e di rappresentanza degli stakeholders privati all'interno del tavolo di concertazione e i problemi di potere, democrazia ed equità di questo strumento di governance del territorio.

\section{Bibliografia}

Albrechts L (2004). Strategic (spatial) planning re-examined, Environment and Planning B: Planning and Design, 31: 743-758; DOI: 10.1068/b3065.

Andrew C., Goldsmith M. (1998). From Local Government to Local Governance: And Beyond? International Political Science Review / Revue internationale de science politique, vol. 19, n. 2: 101-117; DOI: 10.1177/019251298019002002.

Baldi P. (2009). Introduzione. In: Regione Toscana, a cura di, I patti per lo sviluppo locale: uno strumento di governance multilivello (Quaderni della programmazione). Firenze: Giunta Regione Toscana.

Baldi P., Montomoli M.C., Mugnai G. (2008). Strumenti per la governance di area vasta, paper presentato nella XXIX Conferenza italiana di Scienze Regionali. Bari, 24-26 
settembre, 2008. Testo disponibile al sito:

http://www.aisre.it/images/old_papers/Baldi_Montomoli_Mugnai.doc (ultimo accesso 14 novembre 2016 ).

Balducci, A., Bertolini, L. (2007). Reflecting on practice or reflecting with practice?, Planning theory and practice, 8 (4): 530-555; DOI: 10.1080/14649350701664770.

Balducci A., Fedeli V. e Curci F., (2017a). Per guardare oltre la metropoli. In: Balducci A., Fedeli V. e Curci F., a cura di (2017). Oltre la metropoli. L'urbanizzazione regionale in Italia. Milano: Guerini e associati.

Balducci A., Fedeli V. e Curci F., a cura di (2017b). Ripensare la questione urbana. Regionalizzazione dell'urbano in Italia e scenari di innovazione. Milano: Guerini e associati.

Balducci A., Fedeli V. e Curci F., a cura di (2017c). Oltre la metropoli. L'urbanizzazione regionale in Italia. Milano: Guerini e associati.

Balducci A., Fedeli V. e Curci F., a cura di (2017d). Ripensare la questione urbana. Regionalizzazione dell'urbano in Italia e scenari di innovazione. Milano, Guerini e Associati.

Balducci A., Fedeli V., Pasqui G., a cura di (2011). Strategic planning for contemporary urban regions. Farnham (UK): Asghate.

Balz V.E., Zonneveld W.A.M. (2015). Regional design in the context of fragmented territorial governance: South Wing Studio, European Planning Studies, 23,5: 871-891; DOI: 10.1080/09654313.2014.889662.

Barca F. (2009). An agenda for a reformed cohesion policy. A place-based approach to meeting European Union challenges and expectations. Indipendent report. Testo disponibile al sito: http://www.europarl.europa.eu/meetdocs/2009_2014/documents/regi/dv/barca_report_/b arca_report_en.pdf (ultimo accesso 2 marzo 2017).

Bielli M., La Bella A., (a cura di), (1982). Problematiche dei livelli sub-regionali di programmazione. Franco Angeli, Milano.

Bobbio L. (2002). I governi locali nelle democrazie contemporanee. Roma-Bari, Laterza.

Bonomi A. (2016). Nella metamorfosi della crisi vince la grande bellezza, Il sole 24 ore del 3 luglio 2016: 15.

Brenner N. (2003). Metropolitan institutional reform and the rescaling of state space in contemporary western Europe, European Urban and Regional Studies, 10 (4): 297-324; DOI: $10.1177 / 09697764030104002$

Brenner N., Schmid C. (2015). Towards a new epistemology of the urban? City, 19 (2- 3): 151-182. DOI: 10.1080I 13604813.2015.1014 712 .

Censis, a cura di (2013). Rileggere i territori per dare identità e governo all'area vasta. Roma: Censis.

Commissione Europea (2008). Libro verde sulla coesione territoriale. Fare della diversità territoriale un punto di forza, Bruxelles, Commissione Europea. Testo disponibile al sito: http://ec.europa.eu/regional_policy/archive/consultation/terco/paper_terco_it.pdf (ultimo accesso 14 novembre 2016).

Conti S., Salone C., a cura di (2011). Programmazione integrata e politiche territoriali: profili concettuali, esplorazioni progettuali, Contributi di ricerca IRES - Istituto di Ricerche Economico Sociali del Piemonte, 244: 1-81.

Corò G., Dalla Torre R. (2016). Per un auto-governo dello spazio metropolitano. Processi di cooperazione territoriale in Italia, Francia e Stati Uniti, Diritto della Regione, 1. Testo disponibile al sito: http://diritto.regione.veneto.it/?p=2766\&format=pdf (ultimo accesso 14 novembre 2016).

Council of Ministers responsible for Spatial Planning and Territorial Development (2011). Territorial Agenda of the European Union 2020: Towards an Inclusive, Smart and Sustainable Europe of Diverse Regions. Agreed at the Informal Ministerial Meeting of Ministers responsible for Spatial and Territorial Development, Gödöllö, Hungary. 
DIPE (2015). Il partenariato pubblico - privato: normativa, implementazione metodologica e buone prassi nel mercato italiano, Studiare sviluppo, Italy.

EU (2007) Leipzig Charter on Sustainable European Cities. Testo disponibile al sito: http://ec.europa.eu/regional_policy/archive/themes/urban/leipzig_charter.pdf (ultimo accesso 14 novembre 2016).

Fedele M., Moini F. (2006). Cooperare conviene? Intercomunalità e politiche pubbliche, Rivista italiana di Politiche pubbliche, 1: 71-98; DOI: 10.1483/21754.

Fedeli V. (2011) About "Governance" in urban regions: When territory, sovereignity and citizenship do not match. In: Balducci A., Fedeli V., Pasqui G., a cura di (2011). Strategic planning for contemporary urban regions. Farnham (UK): Asghate.

Fernandez E., Rubiera F., (a cura di), (2012). Defining the spatial scale in modern regional analysis. Dordrecht, Springer. DOI: 10.1007/978-3-642-31994-5.

Ferrari G.F., Galeone P., (a cura di), (2010). Città a confronto: le istituzioni metropolitane nei paesi occidentali. Il Mulino, Bologna.

Fregolent, L., Vettoretto, L. (2017) Spazi metropolitani e post-metropolitani nel Veneto contemporaneo. In: Balducci A., Fedeli V. e Curci F., a cura di (2017). Oltre la metropoli. L'urbanizzazione regionale in Italia. Milano: Guerini e associati.

Glassom J., Marshall T. (2010). Regional planning. Abingdon (UK): Routledge.

Gualini E. (2001). Planning and the intelligence of institutions. Asghate (UK), Aldershot.

Healey P. (2010). Making better places. Basingstoke (UK): Palgrave McMillian.

Healey P. (2007). Urban complexity and spatial strategies. London (UK), Rouledge.

Hulst R., Van Montfort A. (2007). Inter-Municipal Cooperation in Europe. Dordrecht, Springer.

Hartman S. e al. (2011). Het adaptieve vermogen/The capacity to adapt. In: H. Ovink e E. Wierenga (Eds) Regio's in verandering. Ontwerpen voor adaptiviteit/Regions in Transition. Designing for adaptivity. Design and politics, 5. Rotterdam (NL), Uitgeverij 010.

Knieling J., Othengrafen F. (2015) Planning Culture. A Concept to Explain the Evolution of Planning Policies and Processes in Europe? European Planning Studies, 23(11): 2133 2147; DOI: 10.1080/09654313.2015.1018404.

Infussi, F. a cura di (2011). Dal recinto al territorio. Milano, esplorazioni nella città pubblica. Milano, Feltrinelli.

Lingua V. (2017). Dalle tattiche alle strategie e ritorno: pratiche di contaminazione nel Regional Design. Urbanistica, 157: 55-60.

Marotta G., Pastena E. (a cura di), (2013). Le città metropolitane. Milano: Cedam.

Messina P. (2011), Policies for strategic territorial development. Inter-municipality association as a form of network governance: the Italian experience. Eastern journal of European studies, 2, 1: 111-128.

Meijers E., Hollander, K. e Hoogerbrugge, M. (2012). A strategic knowledge and research agenda on polycentrism. The Hague (N): EMI - European Metropolitan network Institute.

Paris, M. (2017) Which kind of spatial knowledge supporting smart governance? Two experiences in Castilla y León and Regione Veneto, Territorio, 83: 61-66.

Perulli G. (a cura di), (2014). La città metropolitana. Torino, Giappichelli editore.

Pugalis L., Townsend A.R. (2013) Rescaling of planning and its interface with economic development, Planning Practice and Research, 27 (4): 104-121; DOI: 10.1080/02697459.2012.699236.

Reimer M., GeGmis P., Blotevogel H. (a cura di), (2014), Spatial planning systems and practices in Europe. A comparative perspective on continuity and changes, Abingdon (UK): Routledge.

Rein M., Schon D. (1993). Reframing policy discourse. In: Fischer F., Forester J. (Eds), (1993) The argumentative turn in policy analysis and planning. Londra (UK): UCL Press. 
Servillo L., Lingua V. (2014). The innovation of the Italian planning system: actors, path dependencies, cultural contradictions and a missing epilogue, European planning studies, 22 (2): 400-417; DOI: 10.1080/09654313.2012.752443.

Torfing J. et. al. (2012). Interactive governance. Oxford (UK), Oxford University Press.

Tubertini C. (2014). Area vasta e non solo: il sistema locale alla prova delle riforme. Istituzioni del federalismo, 2: 215-249.

Vandelli L. (2014). Città metropolitane, Province, Unioni e fusioni di Comuni: la legge Delrio, 7 aprile 2014, n56 commentata comma per comma. Rimini, Maggioli editore.

Vandelli L. (2012). Sovranità e federalismo interno. L'autonomia territoriale all'epoca della crisi, Le regioni, 5-6: 845-897. DOI: 10.1443/74020.

Vinci I. (2011). Governance verticale e policentrismo: lezioni e criticità in vista delle nuove politiche di coesione. In Alcozer F. (a cura di). Middlecities. Città medie oltre il policentrismo. Trento-Barcellona: LIStLab Laboratorio internazionale editoriale.

Vino A. (2012). La pubblica amministrazione tra produzione di servizi e produzione di politiche: governance, innovazione, competenze. Dialoghi, 3, maggio 2012:18-31.

Vino A. (1998). Patti territoriali e progettazione istituzionale. In Botta F. (a cura di). Sviluppo, Territorio e Patti. Quaderno n. 14 del Dipartimento per lo studio delle società mediterranee. Università di Bari: Cacucci. 